\title{
Automatic Heart Peripheral Vessels Segmentation Based on a Normal MIP Ray Casting Technique
}

\author{
Charles Florin $^{1}$, Romain Moreau-Gobard ${ }^{2}$, and Jim Williams ${ }^{2}$ \\ 1 Ecole Nationale des Ponts et Chaussees, \\ Champs-sur-Marne, France \\ florin@cermics.enpc.fr \\ 2 Imaging \& Visualization Department, \\ Siemens Corporate Research, Princeton, NJ, USA \\ \{romain.moreau-gobard,jim.williams\}@scr.siemens.com
}

\begin{abstract}
This paper introduces a new technique to detect the coronary arteries as well as other heart's peripheral vessels. After finding the location of the myocardium through a graph theoretic segmentation method, the algorithm models the heart with a biaxial ellipsoid. For each point of this ellipsoid, we compute the collection of intensities that are normal to the surface. This collection is then filtered to detect the cardiovascular structures. Ultimately, the vessels centerline points are detected using a vessel tracking algorithm, and linked together to form a complete coronary artery tree.
\end{abstract}

\section{Introduction}

In the USA, someone suffers a vascular failure every 29 seconds according to the American Federation of Aging Research. The cost of treating congestive heart failure (CHF) resulting from blocked coronary arteries is between $\$ 20$ and $\$ 50$ billion a year for 4.7 million Americans. Computer Tomography (CT) is more precise than echocardiography, but one can claim that no tool is presently available for the detection of heart peripheral vessels in CT. That is the reason why this new technique for heart vessels segmentation is of relevance for a quicker and more accurate diagnosis of CHF. Vessels segmentation allows the early detection of plaques, aneurysms and abnormal configuration of coronary arteries.

In this paper, we propose a heart peripheral vessels reconstruction solution that assumes the existence of a segmented volume representing the heart myocardium. The method is based upon the fact that the vessels are parallel to the heart surface. Therefore, segmenting the heart wall gives an important piece of information about the blood vessels. We consider a multi-stage approach to achieve this task. The first step consists of segmenting the heart, and acquiring the heart wall shell. Then the surface is modeled by a simple geometrical volume, such as a spheroid. In the next step, a ray is cast from each point on the spheroid surface, and the intersected intensities are recorded. During the next step, the ray collection in 3D is used as a pre-segmentation tool. Each vessel crossed by a ray generates a peak of intensity on the ray's profile curve. This is a simple technique to detect voxels belonging to vessels. High-intensity tubular structures in this voxel space can then be used to detect the vessels. During the last step, a full vessel tree is built, using vessel tracking techniques and minimum spanning tree. 


\section{Ray Collection}

\subsection{Heart Segmentation and Distance Map Computation}

We consider a segmentation algorithm driven from the graph optimization technique [2] with a shape constraint. The idea lying behind this "graphcut" segmentation is to minimize an energy function that is defined on a graph, according to the cut of minimum weight. The energy is written as the sum of two terms : $E_{\text {smooth }}(f)$ that imposes smoothness constraints on the segmentation map, and $E_{\text {data }}(f)$ measuring how the label $f$ is adapted to the data:

$$
\begin{gathered}
E(f)=E_{\text {smooth }}(f)+E_{\text {data }}(f), \\
E_{\text {smooth }}(f)=\sum_{p, q \in \text { neighbors }} V_{p, q}(f(p), f(q)), \\
E_{\text {data }}(f)=\sum_{p \in P} D_{p}(f(p)) .
\end{gathered}
$$

$V_{p, q}$ in (2) is the interaction function between the pair of neighboring pixels $\{p, q\}$, and $D_{p}$ in (3) measures how close the label $\mathrm{f}$ is to the pixel p intensity. It is known [2] that such a method provides a global optimal solution for the case of binary valued $f(p)$. There are also a couple of other methods that can be used to isolate the heart, e.g. a model-based segmentation [3] or segmentation algorithms based on level set methods [8] [10].

The segmentation produces a 3D mask (pixels labeled "object" and "background"). The distance map [6] from this surface can provide valuable constraints during the recovery of the peripheral vessels. For instance, as they stay parallel to the surface of the heart, their distance in the distance map varies smoothly. The distance map is computed by parsing the mask twice, in one direction and in the other one, and then filtering each voxel on an edge (object-background) by a $3 \mathrm{D}$ chamfer mask $\mathrm{M}$ and is then used to model the heart by a simpler geometrical object, such as an ellipsoid, in order to flatten its surface easily using cartography algorithms.

\subsection{Modelization of the Shell by a Spheroid}

In the next step, the distance map is used to model the heart wall by an ellipsoid or a biaxial spheroid. Although one may consider a more accurate model like a triaxial ellipsoid, a major drawback is that there is no simple mathematical solution to the $3 \mathrm{D}-2 \mathrm{D}$ projection. The biaxial ellipsoid projection is a well known technique in cartography. Nevertheless, the biaxial spheroid reduces the deformations we could have using a sphere. For a biaxial ellipsoid, of semi-axes length a and $b$, the surface equation is

$$
\frac{x^{2}}{a^{2}}+\frac{y^{2}}{a^{2}}+\frac{z^{2}}{b^{2}}=1
$$

or, in a parametric form: $x=a \cos (\lambda) \sin (\phi), y=a \sin (\lambda) \sin (\phi), z=b \cos (\phi)$ where $\lambda \in[0,2 \pi]$ and $\phi \in[0, \pi]$. In reference to cartography, $\lambda$ and $\phi$ are called longitude and 


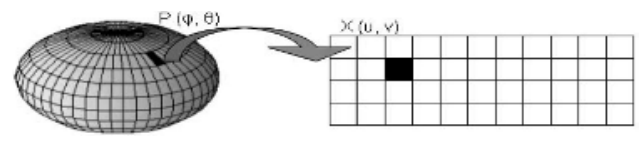

Fig. 1. 3D onto 2D projection, from an ellipsoid onto a plane

latitude respectively. The ellipsoid center is computed as the center of gravity $G$ of all the points located on the distance map isosurface 0 (the heart shell). The large axis $\mathbf{Z}$ is the vector $\overrightarrow{G M}$, where $M$ is the point on the isosurface maximizing the length $\|\overrightarrow{G M}\|$. Similarly, the small axis $\mathbf{X}$ is the vector $\overrightarrow{G N}$, where $\mathbf{N}$ is the point on the isosurface minimizing the length $\|\overrightarrow{G N}\|$. The axis $\mathbf{Y}$ is deduced to have a direct orthogonal base, $B=(G, \mathbf{X}, \mathbf{Y}, \mathbf{Z})$.

Note that the quality of the modeling does not rely on the quality of the segmentation, which makes the method described in this paper independent from the selection of the segmentation algorithm, and robust to noise. Moreover, unlike other organs, one can claim that the heart naturally has the shape of an ellipsoid. From the ellipsoid surface, rays are cast to compute a $2 \mathrm{D}$ view of the heart surface.

\subsection{Ray Casting and n-MIP Projection}

Once the distance map is computed, we cast rays from the ellipsoid, and collect the voxel intensities in a predefined direction and range inside and outside the heart wall. A very similar method is used for visualization purposes solely and applied to the cerebral cortex [7]. The distribution of the nodes on the ellipsoid used to cast rays is recovered through a simple transformation: for each point $\mathrm{P}(\lambda, \phi)$ in 3D-space (Figure 1), the 2D-point $X(u, v)$ is computed according to

$$
\begin{gathered}
{[-\pi, \pi] \times[-\pi / 2, \pi / 2] \longmapsto[0, W] \times[0, H],} \\
(\lambda, \phi) \longmapsto\left(u=\frac{W}{2 \pi} \lambda, v=\frac{H}{\pi} \phi\right) .
\end{gathered}
$$

One could think about casting rays directly from the distance map itself, but in this case, the rays would not be homogeneous enough to be exploitable. Indeed, because of the heart surface irregularities, the rays would miss many vessels. For comparison purposes, we also implemented another solution, based on transverse Mercator projection for a biaxial ellipsoid. The drawback of such a method is that the volume has to be an ellipsoid of revolution, meaning the semi-minor axis is on the polar plane, and the semimajor axes are on the equatorial plane. Examples of unfolded n-MIP view are shown for different patients (Figure 2) with inverted colors. As the intensities have been inverted for the views to look like angiograms, the coronary arteries appear darker, compared to the other heart components. Each pixel I $(x, y)$ on the 2D view is the normalized sum of each intensity, $I_{n}$, of the ray

$$
I(x, y)=\frac{1}{N} \sum_{0<n<N+1} I n
$$




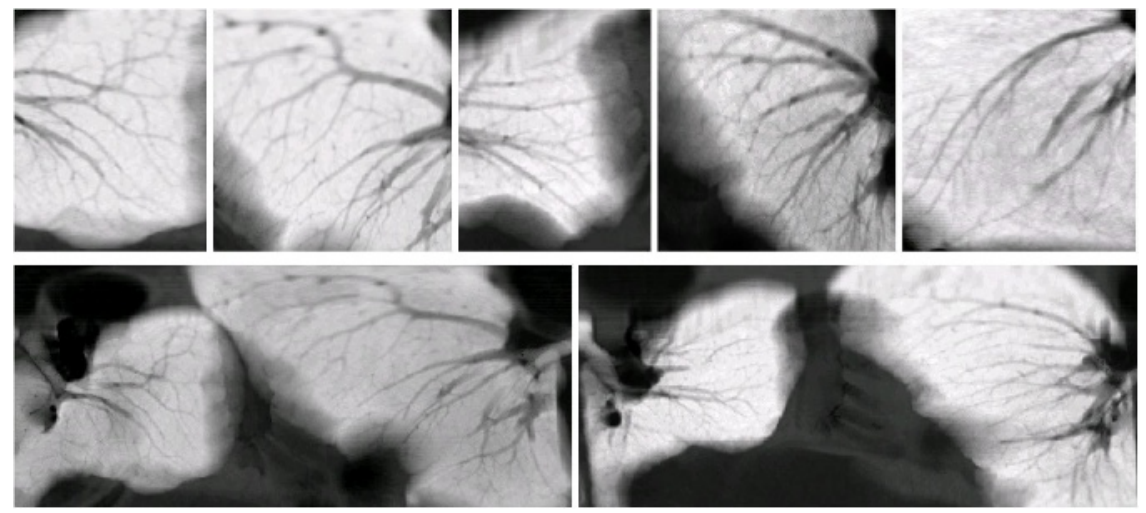

Fig. 2. Unfolded n-MIP (normal MIP) views of heart peripheral vessels for various patients, with inverted colors
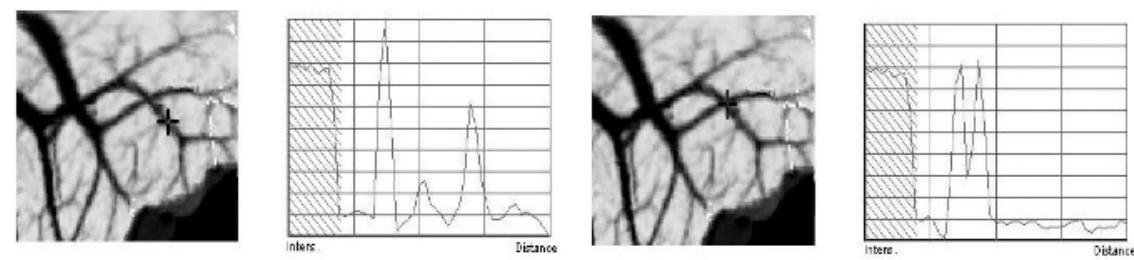

Fig. 3. Profile curves at two different locations on the heart's surface - Myocardium area indicated in crosshatched area

A profile matching technique is used to detect whether or not a ray crosses a vessel structure. As shown in Figure 3, the ray profile curve starts in the heart wall, which is represented as a large, flat, high intensity area. The vascular structures appear as peaks of high intensity, of limited diameter, and at a certain distance from the pericardium.

Next, peaks are processed to determine if they are suitable candidates for a vessel point, according to the following criteria:

1. It respects certain intensity properties (intensity value and peak shape)

2. It is within a certain distance from the heart wall

The intensity peaks, added to the local maximum (a ridge detection [11]), allow a fair detection of the vessels on the rays' profile curve (Figure 3]. In order to detect peaks, we used a zero-crossing of the Laplacian, with the following kernel: [-1 2 -1].

Upon completion of such a procedure, a set of $3 \mathrm{D}$ points that are on a vessel, but not necessarily represent the vessel centerline is available (Figure 4). Such a condition has its origin in the ray-casting effect, as the rays are homogeneously distributed through space. Therefore, the next step will center these candidate points, filter the noise out, and track the detected vessels. 


\section{Heart Peripheral Vessel Detection}

\subsection{Refining the Vessel Candidate Points to Find the Centerlines}

Vessel candidate voxels can be assumed to be next to or within a vessel's lumen. Nevertheless, they are not yet on the vessel's centerline (Figure 4). Furthermore, one can claim that the peak detection could be sensitive to noise (as one can see in Figure 4 ). Therefore, additional processing is required to center these points and to eliminate the noise as well. The refinement operation consists of an eigenvalues analysis [9] for each 3D-point within its corresponding local intensity space neighbors. With

$$
A_{i j}=\frac{\partial^{2} I}{\partial i \partial j},
$$

where $i$ and $j$ are equal to $x, y$ or $z$, and the image intensity function $I$. At point $P$ in space, the Hessian matrix $M$ is defined as

$$
M(P)=\left[A_{i j}\right]_{[i=x . . z, j=x . . z]} .
$$

As the matrix $M$ is defined, symmetric and positive, the computation of the eigenvalues is straightforward. At the center of the vessels, the three eigenvalues $\lambda_{1}, \lambda_{2}$ and $\lambda_{3}$ verify the following equations [1]

$$
\begin{gathered}
\lambda_{1} \approx 0 \\
\lambda_{2} \approx \lambda_{3}<<0 .
\end{gathered}
$$

There are two principal directions given by the eigenvectors. The vector $\overrightarrow{v_{1}}$ associated with $\lambda_{1}$ corresponds to the local vessel orientation, whereas $\overrightarrow{v_{2}}$ and $\overrightarrow{v_{3}}$ define the vessel tangential plane.

For each candidate point $P$, the intensity function on the tangential cut plane defined by $\overrightarrow{v_{2}}$ and $\overrightarrow{v_{3}}$ is filtered by Gaussian functions, $G_{\sigma}$, with increasing variance $\sigma$. The $G_{\sigma}$ minimizing the $L^{2}$ norm of $\left(I-G_{\sigma}\right)$ is kept as model. Then, a potential function $V_{\sigma}$ is built and minimized [5]. $V_{\sigma}$ represents how well a Gaussian vessel model fits for a

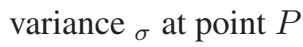

$$
\begin{gathered}
A=\frac{\left|\lambda_{2}\right|}{\left|\lambda_{3}\right|} \\
B=\frac{\left|\lambda_{1}\right|}{\sqrt{\left|\lambda_{3} \lambda_{2}\right|}} \\
S=\sqrt{\lambda_{1}+\lambda_{2}+\lambda_{3}} \\
V_{\sigma}(p)=\left(1-e^{-\frac{A^{2}}{2 \alpha^{2}}}\right) e^{-\frac{B^{2}}{2 \beta^{2}}}\left(1-e^{-\frac{S^{2}}{2 \gamma^{2}}}\right)+\int\left|G_{\sigma}-I\right|^{2}
\end{gathered}
$$

where $\alpha, \beta$ and $\gamma$ are used to control the sensitivity of $V_{\sigma}$ to the diverse ratio A, B and $\mathrm{S}$. This approach is based on an intensity analysis. Other approaches would consist, for instance, in averaging the candidate points over a neighborhood. However, noise and high spacing between the points depending on the density of rays make these methods unsuitable for our application. To account for the high spacing between vessel points, we consider a vessel tracking technique to increase the number of points, and link them together to get the complete coronary arteries tree. 


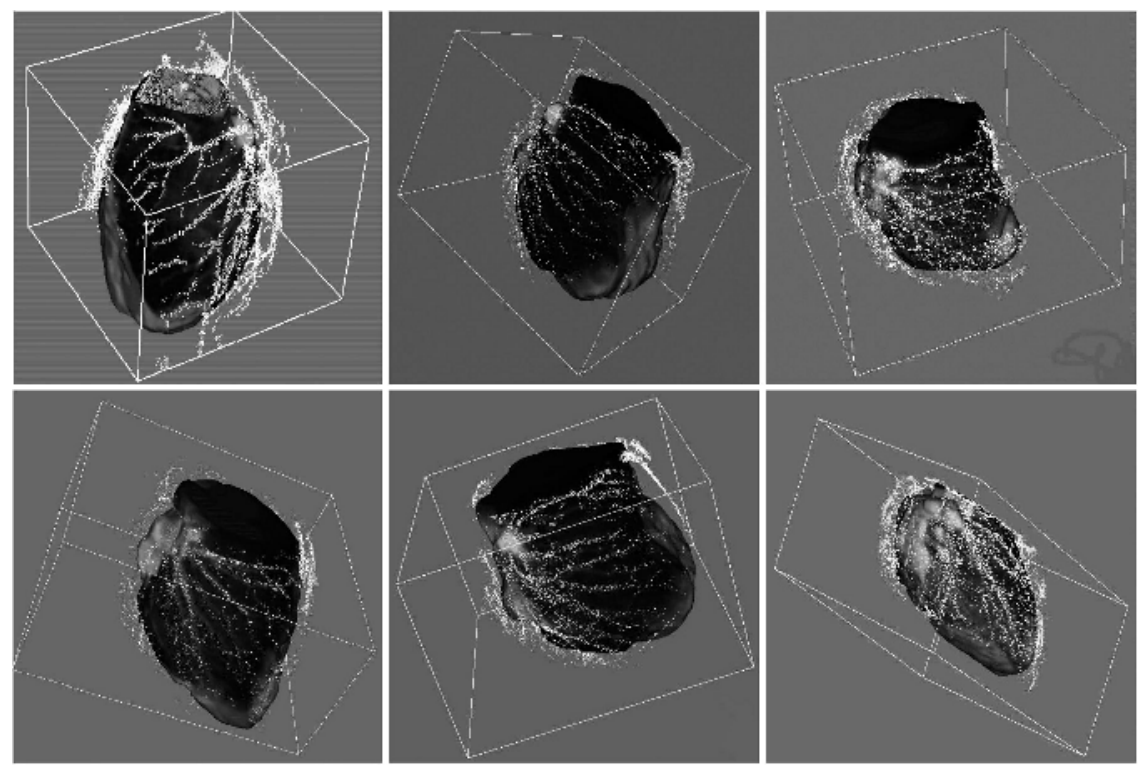

Fig. 4. Vessel Candidates displayed in 3D are not aligned on the vessels centerline

\subsection{Points Densification}

After the eigenanalysis, we assume we have the points, correctly placed, even though their number is not sufficient to track a vessel's centerline. Thus, in order to place more points, a simple vessel tracking operation is performed for each 3D candidate point, under the conditions of linear intensity variation, consistent distance from the segmented heart wall, and consistent orientation. In other words, from a point resulting from the eigenanalysis, we build another point and link it to the previous one, thus tracking the vessel. The speed vector $\overrightarrow{v_{t}}$ is the weighted sum of two vectors, the speed vector $\vec{v}_{t-1}$ and the local orientation vector $\overrightarrow{v_{1}}$ from 7

$$
\overrightarrow{v_{t}}=\lambda \times \overrightarrow{v_{t-1}}+(1-\lambda) \times \overrightarrow{v_{1}}
$$

where $\lambda \in[0,1]$. This speed vector, as well as intensity and distance to the heart, has to be homogeneous. We detected edges through a Laplacian analysis:

$$
\int|\vec{\nabla} I \overrightarrow{d l}|<I_{\text {coronary }}-I_{\text {exterior }} .
$$

If such an inequality is not satisfied for the distance function $D$ and the image intensity $I$, an edge has been detected, and the tracking operation stops. Otherwise this tracking operation is performed until we reach the end of the vessel, or we can link all the chains to another point. At this stage, the candidate points are centered and homogeneously distributed on the centerline of the vessel. They are linked by a minimum spanning tree 

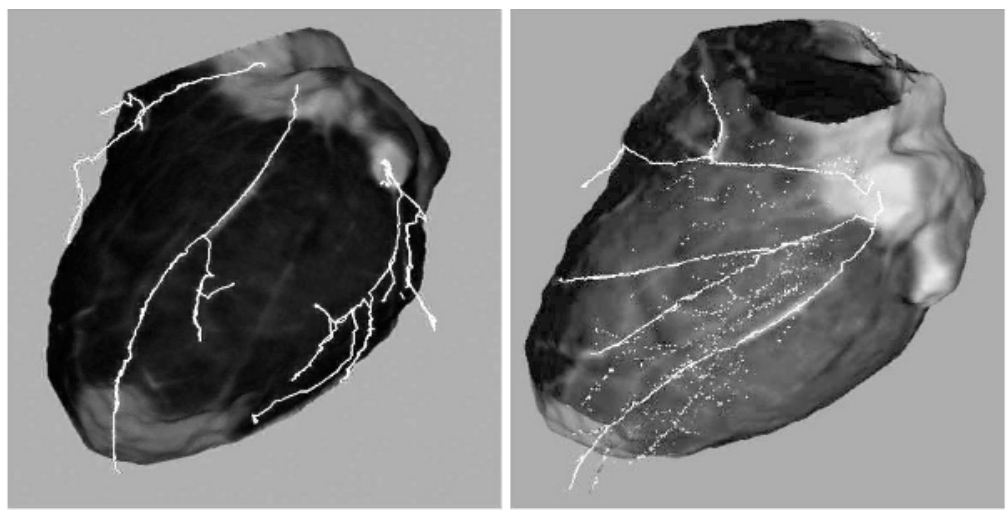

Fig. 5. The points on the vessels centerline are linked together

[13] algorithm, minimizing the following cost function $E$ between two points $P_{1}$ and $P_{2}$

$$
E_{P_{1} P_{2}}=\left|D\left(P_{1}\right)-D\left(P_{2}\right)\right|^{2}+\left|I\left(P_{1}\right)-I\left(P_{2}\right)\right|^{2}+\left\|\overrightarrow{P_{1} P_{2}}\right\|^{2}+\left|\overrightarrow{v_{1}}\left(P_{1}\right) \cdot \overrightarrow{v_{1}}\left(P_{2}\right)\right|,
$$

and result in the peripheral vessel's centerline (Figure 5).

Other algorithms are available to group together connect components [4] [14] [15]. These algorithms will be implemented in further studies. An interesting approach is based on a supervised region expansion [12].

\section{Results and Conclusion}

A heart peripheral vessel segmentation algorithm was presented in this paper, based on a ray filtering algorithm. In a step-by-step approach, the heart is first segmented using an algorithm based on graph cuts and geodesic surfaces, and a 3D distance map is computed out of the segmentation's output. Then, from this distance map, the biaxial ellipsoid modeling the heart is computed, and rays are cast from its surface toward the heart. Then, candidate points likely belonging to vessels are detected along the n-MIP rays' profiles. Finally, the vessel's centerline is tracked from the candidate points. This method can be used, for example, to visualize the full peripheral vessel tree, and detect plaques and aneurysms. Once segmented, the vascular structures can be unfolded, and the quantification of the stenosis and the aneurysms is straightforward. The segmentation results support efficient reporting by enabling automatic generation of overview visualizations, guidance for virtual endoscopy, generation of curved MPRs along the vessels, or cross-sectional area graphs. Moreover, applied to CT data sets, this algorithm detected vascular structures (Figure 5) quickly enough for industrial applications (60 seconds for a bi-processor $900 \mathrm{MHz}, 1 \mathrm{~GB}$ RAM). Beside time constraints, the main advantage of this method compared to techniques relying on front propagation is its robustness to noise. 
We are currently working on a hybrid front propagation algorithm that combines the above-mentioned technique with a multiscale vesselness measure. Moreover, another surface to model the heart is under investigation, to minimize the distortions introduced by the ellipsoid.

Acknowledgments. The authors would like to thank Christophe Chefdhotel for his help with the minimum spanning tree algorithm, Yuri Boykov for his precious help with his Graphcut segmentation algorithm, and the reviewers for their advice.

\section{References}

1. Stephen Aylward, Elizabeth Bullitt, Stephen Pizer, and Charles Chung. Tubular objects in 3d medical images: Automated extraction and sample application. In 1999 Radiology Research Review, 1999.

2. Yuri Boykov, Olga Veksler, and Ramin Zabih. Fast approximate energy minimization via graph cuts. In ICCV, pages 377-384, 1999.

3. T.F. Cootes, C.J. Taylor, D.H. Cooper, and J. Graham. Active shape models: Their training and application. CVIU, 61(1):38-59, January 1995.

4. T. Deschamps and L.D. Cohen. Geometric Methods in Bio-Medical Image Processing, chapter Grouping connected components using minimal path techniques. Mathematics and Visualization. Springer, 2002.

5. Alejandro F. Frangi, Wiro J. Niessen, Koen L. Vincken, and Max A. Viergever. Multiscale vessel enhancement filtering. Lecture Notes in Computer Science, 1496:130-??, 1998.

6. S. F. Frisken Gibson. Calculating the distance map for binary sampled data. Technical Report TR99-26, Mitsubishi, 1999.

7. Junfeng Guo, Alexandru Salomie, Rudi Deklerck, and Jan Cornelis. Rendering the unfolded cerebral cortex. In MICCAI, pages 287-296, 1999.

8. M. Kass, A. Witkin, and D. Terzopoulos. Snakes: Active contour models. In Proc. of IEEE Conference on Computer Vision, page 259-268, London, England, 1987.

9. K. Krissian, G. Malandain, N. Ayache, R. Vaillant, and Y. Trousset. Model based detection of tubular structures in 3d images. Computer Vision and Image Understanding, 80(2):130-171, November 2000.

10. M. Leventon, O. Faugeras, and W. Grimson. Level set based segmentation with intensity and curvature priors. In Workshop on Mathematical Methods in Biomedical Image Analysis Proceedings (MMBIA), pages 4-11, June 2000.

11. T. Lindeberg. Edge detection and ridge detection with automatic scale selection. Int. J. of Computer Vision, 1996.

12. Cristian Lorenz, Steffen Renisch, Thorsten Schlathlter, and Thomas Bulow. Simultaneous segmentation and tree reconstruction of the coronary arteries in msct images. In SPIE International Symposium on Medical Imaging, volume 5031, 2003.

13. Seth Pettie and Vijaya Ramachandran. An optimal minimum spanning tree algorithm. $J$. ACM, 49(1):16-34, 2002.

14. G. Shechter, F. Devernay, A. Quyyumi, E. Coste-Mani 'ere, and E.R. McVeigh. Three-dimensional motion tracking of coronary arteries in biplane cineangiograms. IEEE Trans. Med. Imaging, 22(4):493-603, April 2003.

15. Alexander Vasilevskiy and Kaleem Siddiqi. Flux maximizing geometric flows. IEEE Trans. Pattern Anal. Mach. Intell., 24(12):1565-1578, 2002. 